\title{
A Study on Internet of Things Technology and Its Application in Intelligent Logistics
}

\author{
Limei Duan \\ Kunming Metallurgy College, Kunming, China
}

Keywords: Internet of Things; intelligent logistics; structure; Internet

\begin{abstract}
The Internet of Things is called the third wave of the world's information industry following the computer and the Internet. With the Internet of Things being formally included in one of the country's five most important emerging strategic industries, the Internet of Things has created an upsurge in China and provided the technical prerequisites for the realization of intelligent logistics. Based on the author's learning and practical experience, this paper first analyzed the basic components of the Internet of Things system and then introduced the structure of the Internet of Things. Finally, this paper proposed the application of the Internet of Things technology in the intelligent logistics system. The intelligent logistics system centered on the Internet of Things technology integrates the warehousing, transportation, distribution and packaging in the logistics industry chain and it integrates radio frequency technology, wireless sensor network technology, logistics real-time tracking technology, networking and communication technology. The logistics industry has been greatly improved and optimized and it is in line with the new trends of intelligent, automatic and networked development of the logistics industry.
\end{abstract}

\section{Introduction}

The operational and economic efficiency of the logistics supply chain system depends on the efficient and smooth connection of a series of links of cargo handling, packaging, transportation and storage, as well as real-time monitoring and management within the links. The traditional logistics management cannot collect the above data in real time, completely and accurately. It is difficult for the tradition one to monitor the logistics supply chain process and it is impossible to achieve the improvement of logistics efficiency and economic efficiency. In contrast, intelligent logistics based on the Internet of Things technology is characterized by the transparency of process data in the logistics supply chain, real-time logistics monitoring and intelligent decision-making in supply chain management. It can achieve efficient automation, intelligent supervision of operations as well as safe and reliable logistics transportation in all aspects of the logistics supply chain. By excavating the needs of logistics customers, real-time monitoring of the logistics transportation process and optimizing the management of commodity inventories, logistics management automation, efficient and convenient logistics operation and customer-centred philosophy can be realized.

\section{Basic components of the Internet of Things system}

\subsection{Hardware platform of Internet of Things}

The Internet of Things is a data-centred and application-oriented network. It mainly implements functions such as information awareness, data processing, data transmission and decision-making support. The hardware platform can be composed of a sensor network, a core bearer network and an information service system. The schematic diagram of the hardware platform of the system is shown in Figure 1. Among them, the sensor network includes sensing nodes (data collection and control) and the end network (sink nodes and access gateways); the core bearer network is the basic communication network of the Internet of Things business; the hardware facilities of the 
information service system are mainly responsible for processing information and supporting decision-making.

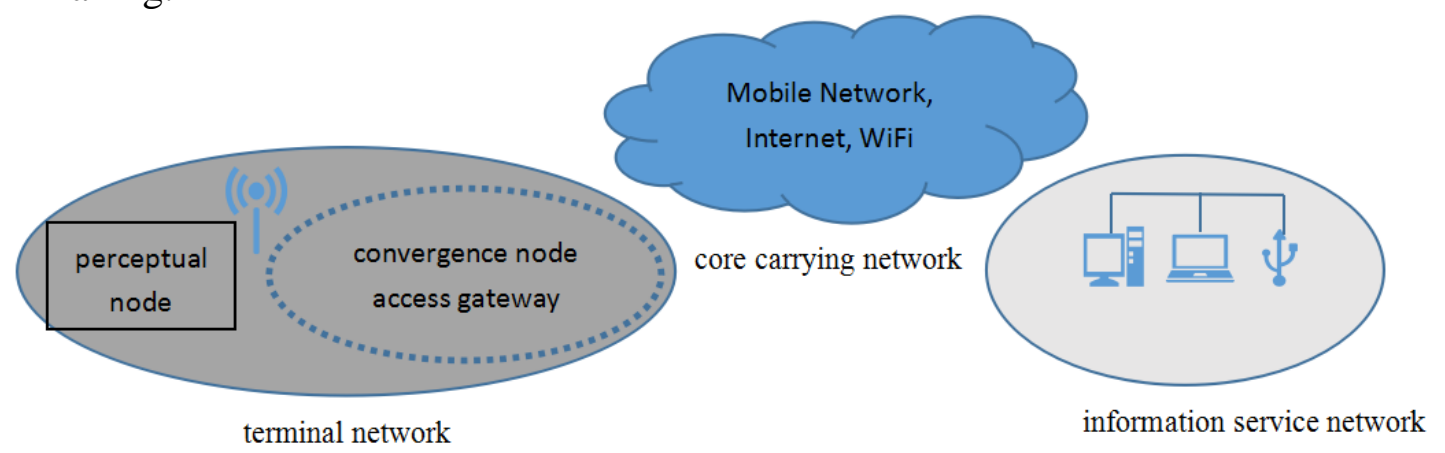

Figure 1 Schematic diagram of the hardware platform of the Internet of Things

(1) Sensing nodes. The sensing node is mainly composed of various types of data acquisition modules, such as sound sensors, temperature sensors, pressure sensors, vibration sensors, twodimensional code readers and RFID readers to complete the initial data acquisition and device control of the Internet of Things applications.

(2) The end network. The end network is to access the network, including sink nodes and access gateways. It completes networking control and data aggregation in the end sensing nodes or completes the forwarding of data to the sensing nodes.

(3) Core bearer network. There may be many kinds of core bearer networks, which are mainly responsible for the data communication tasks between the information service system and the access network. According to the specific application needs, the bearer network can be a public communication network, such as $2 \mathrm{G}$, $3 \mathrm{G}$ and $4 \mathrm{G}$ mobile communication network, WiFi, WiMAX, Internet, private enterprise network, or even a new communication network dedicated to the Internet of Things.

(4) Information service system. Information service system of the Internet of Things is mainly composed of various application servers (including database servers) and other hardware facilities. It also includes user equipment (such as PCs and mobile phones) and clients. It is mainly used for convergence, aggregation, conversion, and analysis of collected data, adaptation to user presentations and event triggering.

\subsection{Software platform of Internet of Things}

The software platform is the neural network of the Internet of Things. Different types of Internet of Things have different software system platforms. In general, the software platform of Internet of Things is built on a layered communication protocol system, which usually includes data-aware system software, middleware system software, network operating systems (including embedded systems) and information management systems for the management and information center of the Internet of Things.

(1) Data-aware system software. The data-aware system software mainly completes the collection and processing of items' EPC codes and the identification of items. It is mainly composed of articles produced by enterprises, including electronic tags of items, readers, controllers, sensors and item codes (EPC).

(2) Middleware system software of Internet of Things. The middleware is an application software located between the data-aware facility (reader) and background application management software. The middleware provides a series of computing and data processing functions for the Internet of Things application. The main task is to capture, filter, converge, compute, demodulate, proofread, manage, transfer and store data collected by the sensing system..

(3) Network operating system. The Internet of Things interconnects any item in the physical world through the Internet. Each item in the Internet of Things becomes an "intelligent product" with dynamic information. The Internet of Things can promote the synchronization of logistics and 
information flow of items, thus building an article information sharing platform with fast and efficient network information and cloud computing.

(4) Information management system of Internet of Things. Similar to Internet network management, the Internet of Things also needs to be managed. It is an information management system software for Internet of Things of management organizations (including the enterprise information management center of Internet of Things, the national information management center of Internet of Things and the international information management center of Internet of Things).

\section{The structure of the Internet of Things}

The Internet of Things is a huge system. It is mainly composed of an information acquisition system, an information transmission system and an information processing system. The information acquisition system is mainly responsible for positioning, sensing and collecting information. It is composed of a large number of sensors and RFIDs. It can be achieved by information sensing technology. The key technology includes radio frequency identification technology, information location positioning technology, sensor technology, multimedia information acquisition technology and two-dimensional code technology. The information transmission system is the extension based on the Internet technology. It can aggregate and sense data and can transmit and process the data of the Internet of Things. The covering technology includes the Internet, wireless networks and satellite communications. In particular, mobile communication technology developed in recent years plays a key role in mobile Internet of Things communications. The information processing system is the technology used for remote processing and using of date of the Internet of Things. It can be realized through cloud computing, artificial intelligence, embedded systems, knowledge discovery and data mining technology, multimedia and virtual technology. The Internet of Things technology includes multiple levels of technology and involves multiple application areas. After the key technology is popularized, they will mainly be used in sensing devices of animals, plants, machines, articles, electronic labels and matched interface devices, especially for the sensing device of the logistics distribution system. This will change the traditional logistics system and model, enhance the optimization and upgrading of the industry and drive the innovative development of economy. In recent years, the National Development and Reform Commission made a clear orientation to the country's new strategy. Intelligent logistics is one of the applications of the Internet of Things in the logistics distribution system.

The intelligent logistics system is the organic integration of the key technology of the Internet of Things and the logistics delivery system. It integrates the sensing technology, computer technology, Internet technology, decision-making technology of the Internet of Things to achieve comprehensive, efficient, intelligent and friendly logistics operation with the automatic sensing of object information, automatic processing of information and intelligent decision-making. It also make the logistics from the supply side to the demand side with flowing intelligent monitoring, so as to reduce the the logistics costs of the supply side and maximize profits. Intelligent logistics embodies the comprehensive informationization process of information technology and logistics supply chain technology in different aspects of products, which provides a favorable platform for enterprises and customers to realize information communication and decision-making support. It is the best way to achieve rational use and allocation of resources. The core technology of the Internet of Things can realize the physical communication on the basis of the Internet and it is complex. Like the computer network structure, it must be designed in layers. Its core functions are information awareness, network transmission and application interfaces. To realize the functions of the Internet of Things, at least three layers need to be applied, including the information sensing layer, the network transmission layer and the data processing layer. The following discussion is about the three-layered structure of the Internet of Things and its implementation technology. The technical structure is shown in Figure 2. 


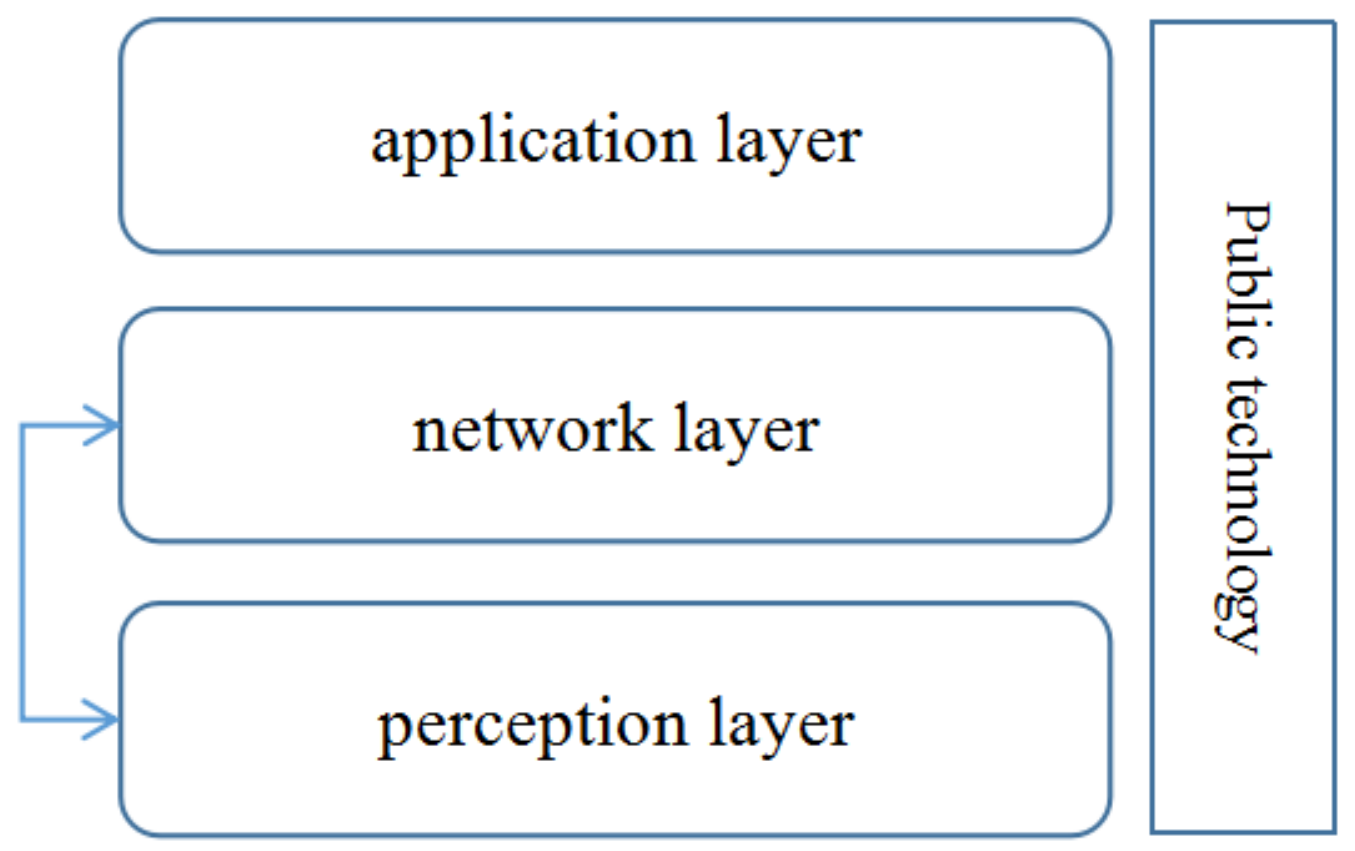

Figure 2 Structure of the Internet of Things

The information sensing layer is mainly used to collect physical events and data in the real world. Its function is to identify objects, collect information and identify data status. At the same time, it transforms the collected data from actual physical data to virtual digitally processed data through corresponding technology and means. The main function of the network transmission layer is to use existing network technology and communication technology to achieve reliable and efficient transmission of information collected by the sensing layer. The industry characteristics of the Internet of Things are mainly reflected in the application areas. Its functions are achieved by application layer technology. This is the highest level of the Internet of Things. Currently, it has a wide range of applications in areas such as intelligent transportation, power, home and logistics. By integrating the needs of different industries and customers, it is constructing an operating platform for different applications to achieve intelligent, portable and accurate information service. The main contents involved in the public technology level include identification analysis, security technology, QoS management and network management. These public technologies are the technical guarantees for the operation of the Internet of Things system.

\section{Application of Internet of Things technology in intelligent logistics system}

\subsection{Sensing technology}

The information-aware layer is located at the lowest end of the structure of the Internet of Things. The collection of data is the basis of the information system and the data is acquired through the sensing layer, which is finally transformed into useful decision-making data. The sensing layer mainly implements the marking and identification of information. There are many key technologies such as sensor technology, automatic radio frequency (RFID), near field communication (NFC) and system positioning (PGS) to track, monitor and locate the sensing objects. Automatic sensing RFID technology is mainly applied in the modern intelligent logistics system. RFID tags and smart RF terminals are widely used in some warehouse distribution centers, which effectively promotes the rapid development of intelligent logistics.

\subsection{The design of intelligent logistics system}

\subsubsection{Requirements analysis of system function}

The main function of intelligent logistics system based on RFID technology is to achieve the 
collection of information on the items with RFID technology. In the entire logistics process, the system can achieve the effective tracking and positioning of transport vehicles, and realize intelligent management in the storage management. Therefore, the following functional requirements can be analyzed: (1) Collection of basic information of items. Basic information collection of articles includes source, origin, raw material, type and brand. The information needs to be written in electronic tags via an RFID scanner; (2) Transmission of basic information. The collected data is transmitted to the central database through the network system for query; (3) Registration and login. The information must be registered before query and entry in the system. Besides, necessary identity authentication needs to be conducted to ensure the authenticity and integrity of the commodity information and information transmission; (4) Data query. Consumers can inquire about the goods through the terminal, including the manufacturer, origin, raw materials, categories, brands and other related attributes; (5) Distribution management. Distribution management is mainly to conduct effective classification and distribution based on the location and type of goods; (6) Tracking and positioning. In the management of transport vehicles, the basic information for vehicles is firstly registered in the system. In the process of product distribution, the system updates information in accordance with the use of vehicles, automatically allocates vehicles, and specifies the optimal route. Vehicles can be tracked in real time through GPS during transportation; (7) Information management. It is mainly the effective management of commodity tracking information. Administrator can type in, modify and delete commodity information. First of all, the information provided by the merchant and the customer will be submitted to the administrator with an electronic tag. After the administrator has typed it in the system, it will enter the central database and be ready for inquiry at any time. (8) System maintenance. System administrators manage the system in groups, including super administrators and ordinary administrators. System management and maintenance can be realized with the allocation of corresponding rights. In addition to the above functional requirements, it also involves some nonfunctional requirements, such as how to ensure the reliability and integrity of information transmission, how to achieve convenient queries, how to update data in real time and how to realize efficient mobility without operating systems and hardware systems as far as possible.

\subsubsection{System structure}

According to the analysis of system function requirements, the functions of the intelligent logistics system based on the Internet of Things model mainly include information collection subsystem, data query subsystem, logistics distribution and management subsystem, user system management subsystem, information transmission subsystem, and system operation and maintenance subsystem. Its overall functional structure can be seen in Figure 3:

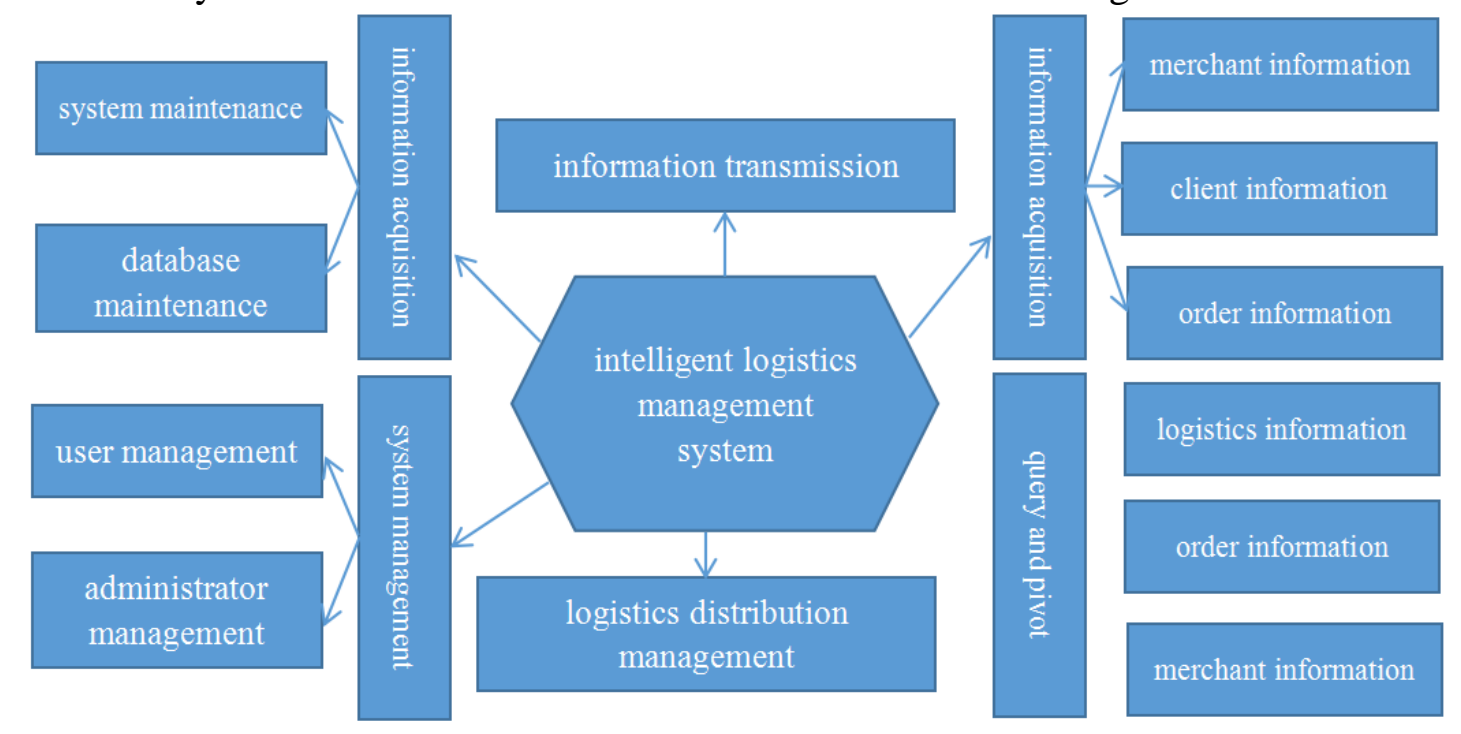

Figure 3 Overall functional structure of intelligent logistics management system 


\subsubsection{Design and realization of key subsystems}

1) Information collection module

The information collection module is mainly composed of the basic information of the commodity, the merchant, the customer and the order. Commodity information mainly includes information on the raw materials, origins, classifications, brands, and manufacturers of commodities, and it is registered in the system with electronic tag after scanned by RFID. Merchant and customer information. Merchant information mainly includes their names, territories, properties, scales and business types; customer information is mainly a unique ID number after the customer registers the system, including basic personal information which can be modified by the individual. The customer can also cancel their personal accounts. Order information. After the customer logs in the system with the account assigned and identified by the system, the customer can view the transaction records among individuals, logistics companies and businesses at any time. If the user logs out, related transaction records will be deleted.

2) Logistics distribution module

Logistics distribution management includes warehouse management, distribution management and vehicle management. According to the actual needs, it can realize the registration of in-put and out-out of storage for goods, the real-time data update of the storage of goods, the distribution and management of vehicles and the tracking and positioning functions in the transportation.

3) Logistics distribution management module

The logistics distribution management module includes inventory management, distribution management and vehicle management. It can realize real-time updates of in-put and out-put goods from warehouse, status of logistics vehicles and dynamic tracking and monitoring in the distribution process with RFID scanning and GPS positioning technology. Storage management is the main aspect of commodity inventory management. Figure 4 shows the in-put and out-put process of commodities.

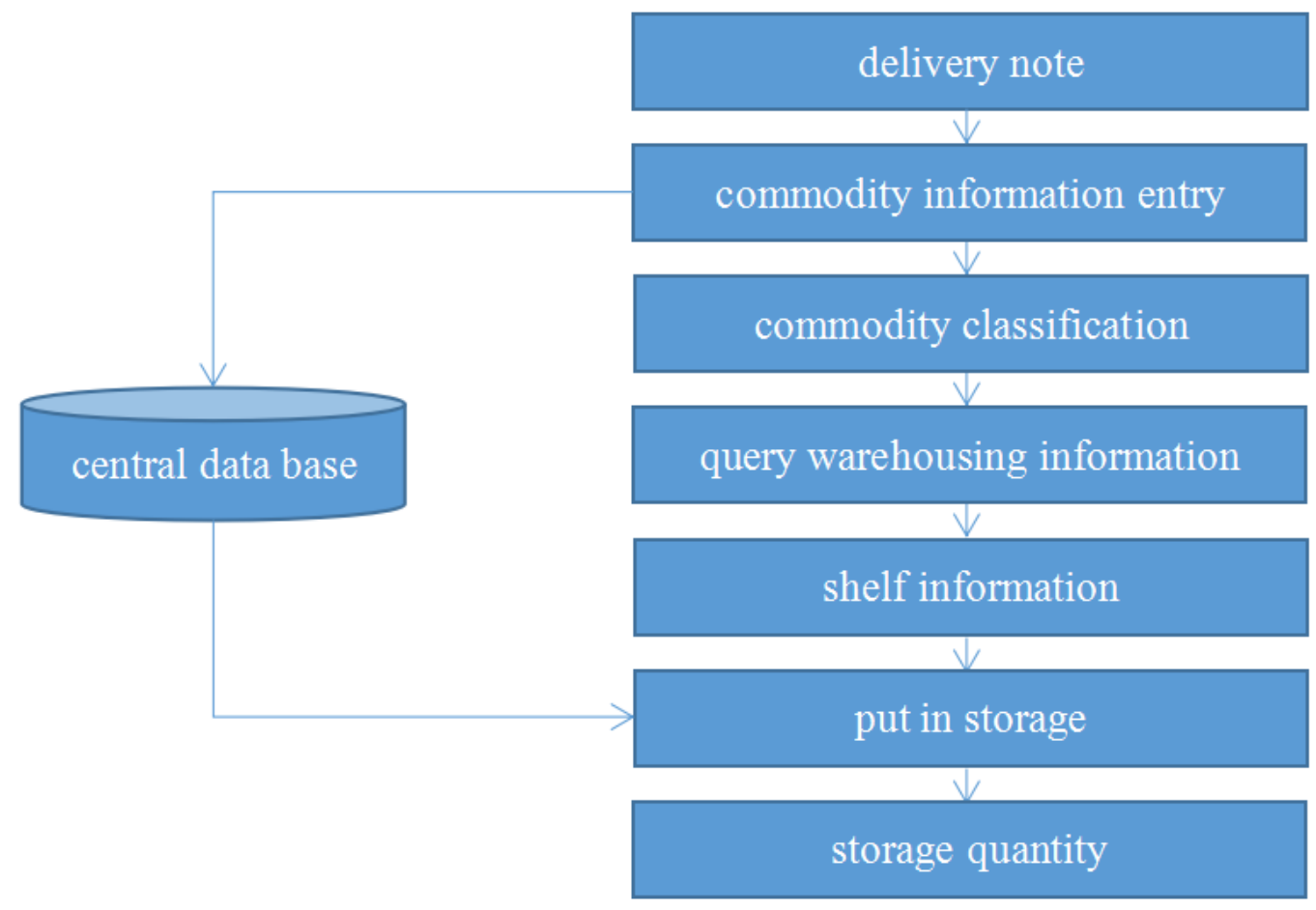

Figure 4 Storage process

The basic information of the goods is transmitted to the central database through the information collection system. Each commodity has its EPC code which is written into the corresponding electronic tag as the unique identifier of the product. At the same time, the warehouse management system specifies a corresponding position. At the time when goods are put in storage, the RFID scanning device transmits the information to the main control system. The system can query the 
storage dynamic information. Using the data binding associates the information of goods that are put in the warehouse with the storage information and fills in the form. The storage administrator can search the commodity list in the computer system. At the same time, according to the basic information of the form, the warehouse manager determines the designated shelves and positions for the incoming vehicles and goods to be stored in the warehouse. When the vehicle passes through the storage barrier, the basic information on the vehicle will be perceived by the reader installed at the crossing. The electronic screen will display the corresponding shelf number and orientation. The corresponding shelf light is turned on. After the warehouse manager confirms it, it will be automatically placed on the shelf. When the commodity approaches the shelf, the reader senses the basic information of the commodity and registers it in the background database. Then the shelf indicator goes out and the system updates the central database and generates a storage order. The storage process is completed.

The delivery process of cargo from storage is basically opposite of storage, as shown in Figure 5. First, computer system automatically generates form of delivery order form. Warehouse managers scan the form to verify the information goods out of the warehouse and query vehicle information through the central database. If there are free vehicles, the manager proposes dispatching requests in a timely manner. When the scheduled vehicle travels to the warehouse where the goods are located, the crossing sensor device senses the vehicle information and records the vehicle storage information. Then, the electronic screen displays the shelf number and position information of the goods. When the vehicle arrives at the corresponding shelf, the piler takes off the goods and loads them in the vehicle. Finally, the goods and vehicle information is automatically bound and the database is updated. When the vehicles with goods pass through the exit port, the system automatically generates a delivery order and the delivery process of cargo from storage is completed.

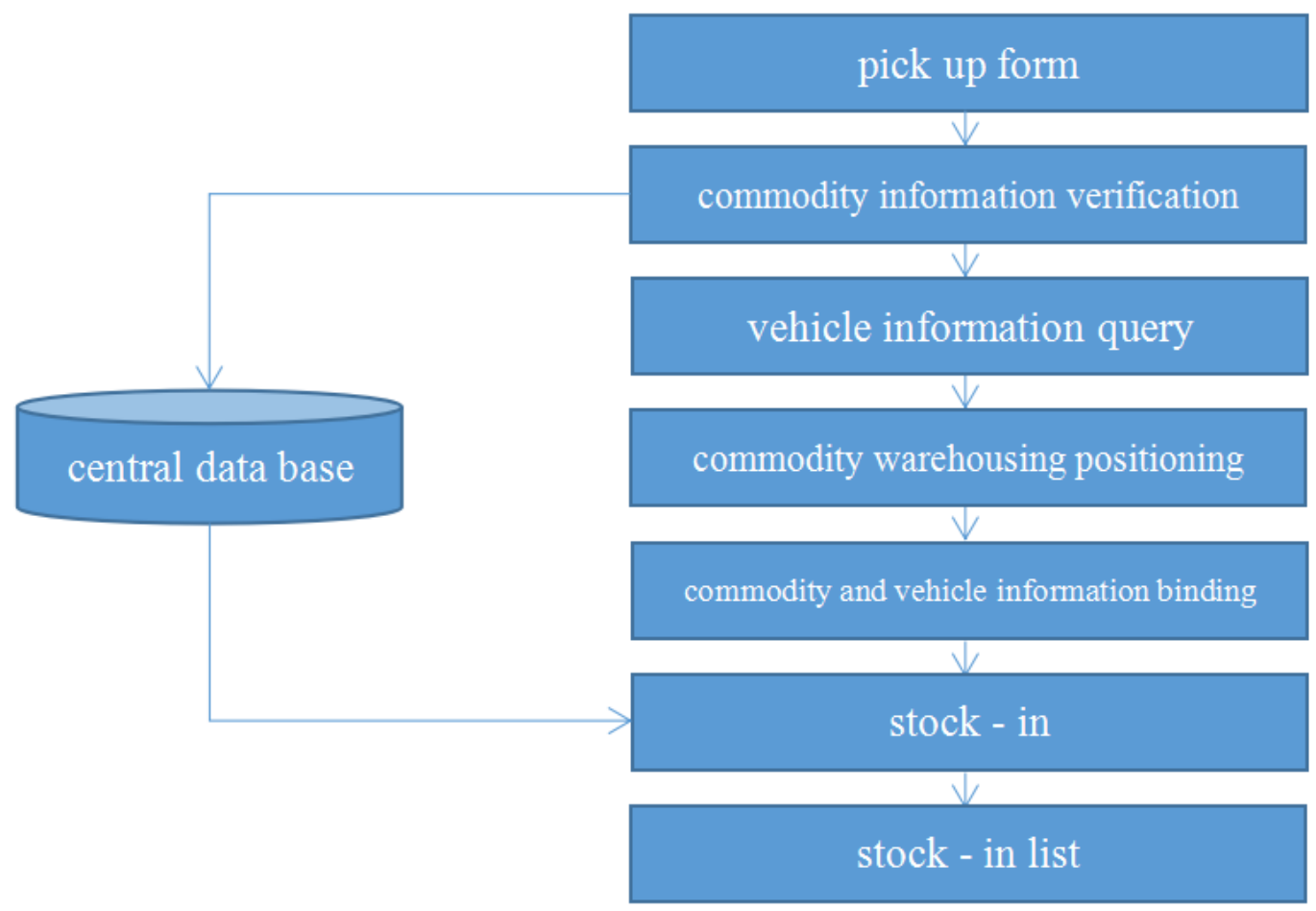

Figure 5 Delivery process of cargo from storage

\subsubsection{System management module}

The system management subsystem includes functions of administrator management and user management. After the administrator has been assigned rights, they can assign and manage the entire system. User management is assigned corresponding rights based on the different roles of users. It needs the system registration, the basic user information recording, single sign-on and user identity authentication. 


\subsubsection{System operation and maintenance module}

The system operation and maintenance module includes the functions of system operation and maintenance and database maintenance. System maintenance involves the setting of system parameters, and backups and updates of system files to ensure the normal operation of the system. The database maintenance function mainly means to manage the central data in real time, ensure the safe and reliable use of data and increase the disaster recovery function simultaneously to improve the stability of the system.

\subsubsection{Data query module}

Data query can realize the function of querying commodity information, logistics information and vehicle information with the WEB client. In addition to the above major systems, there are information transmission systems and report printing systems.

\section{Conclusion}

The Internet of Things is the latest network and is a new epoch-making revolution in science and technology. The application of the Internet of Things technology will provide new challenges and development opportunities for the development of logistics industry in China, especially the intelligent logistics. The operation model of logistics will undergo tremendous changes. However, we should also see that the application of the Internet of Things is still in its infancy. To fully meet the requirements of intelligent logistics, there are still technical problems such as problems in huge investment, technical standards, network structure and protocols, security and privacy, and terminals. It also needs pioneering research.

\section{References}

[1] Lin Ke, Tan Hua, Yang Shaolong, Huang Yue, Li Ying. A Study on the Design of Public Service Platform of Intelligent Logistics Based on Internet of Things Technology [J]. Telecommunications Technology, 2014, (10): 78-82.

[2] Zhou Yue'e. A Study on the Design of Modern Agricultural Intelligent Logistics System Based on Internet of Things Technology [J]. Chinese Journal of Agricultural Mechanization, 2014, (05): 256-260.

[3] Le Mengde. A Study on the Design and Implementation of Intelligent Logistics Supervision System Based on Internet of Things Technology [J]. Market Modernization, 2014, (08):50-51.

[4] Luo Jian. A Study on the Intelligent Logistics System Design for Internet of Things [J]. Automatic Instruments, 2013, (10): 48-50.

[5] Yang Mi, Wang Mingyu, Liu Shuzhen. A Research on Intelligent Logistics Based on Internet of Things [J]. China Business and Trade, 2013, (12): 113-114.

[6] Yang Fan, Xiao Yan. A Research on Advantages of Intelligent Logistics Development and Its Business Model [J]. Comprehensive Transportation, 2013, (04): 27-30.

[7] Yu Shanshan, Wang Sifeng. An Analysis and Design of Intelligent Logistics System Based on Internet of Things [J]. Software, 2012, (05): 6-8.

[8] Pan Wenjun. An Analysis of the Relationship between Internet of Things and Modern Logistics Management [J]. Hoisting and Transport Machinery, 2012, (02): 37-42.

[9] Li Xia. A Discussion on Intelligent Logistics in the Era of Internet of Things [J]. Modern Business, 2011, (26): 9+8.

[10] Chen Fengzhao, Jiang Daihong. A Study on Design and Implementation of Intelligent Logistics Distribution System Based on Internet of Things [J]. Microelectronics and Computers, 2011, (08): 19-21. 
[11] Huang Zhiyu, Yan Qichun, Chen Dengfeng. A Research on Intelligent Logistics Warehousing System in Internet of Things [J]. Automatic Instruments, 2011, (03): 12-15.

[12] Dai Dingyi. A Study on Internet of Things and Intelligent Logistics [J]. China Logistics and Purchasing, 2010, (08): 34-36. 